\title{
RESEARCH INTO FUNCTIONAL EQUATIONS ARISING FROM MATHEMATICAL MODELING OF CRITICAL STATES OF HETEROGENEOUS SOFT LAYERS
}

\author{
V. L. Dilman ${ }^{1}$, dilman49@mail.ru, \\ D. A. Trunova ${ }^{1}$, jessica192@mail.ru. \\ ${ }^{1}$ South Ural State University, Chelyabinsk, Russian Federation.
}

\begin{abstract}
We obtained the classification of solutions to a functional equation arising from the research into mathematical models of critical states of the plastic layer. The layer is exposed to a tensile stress under conditions of plane deformation. The function of the layer heterogeneity depends presumably on two variables. We demonstrated how the research into the mentioned mathematical models can be reduced to the solution of some nonlinear systems of ordinary differential equations under the conditions of separating the variables for tangent stress and for the heterogeneity function.
\end{abstract}

Keywords: soft layer, stress state, hypothesis of variables separation, systems of nonlinear differential equations, functional equations.

\section{Introduction}

Normally the stresses on the contact surface between the plastic layer and the compressive surfaces are unknown. To define the tensile (compressive) force, it is necessary to solve the inverse boundary problem of defining normal stresses on the contact surface. Various mathematic models of the stress state of a plastically deformed body at compression or tension are based, as a rule, on the differences of technological processes. At compression, what is important is the presence or absence of sliding of contact surfaces as well as the value of the friction factor. At compression and tension of the welded joints, the characteristics of the model are influenced by the deformation of the contact surfaces as well as by involvement of the more solid area in the process of plastic deformation. The suppositions applied quite often take the form of limitations over the function classes in which the solution is being looked for $[1,2]$. The hypothesis of the variables separation for tensile stresses has proved to be effective [1 -4].

$$
\tau_{x y}=X(x) Y(y)
$$

In practice, not only the whole joint but also its soft layer is heterogeneous. The basis of the mathematical model is the approximate [ $1-5]$ system of equations of the plastic equilibrium taking the following form for the heterogeneous layer $[1,2,6,7]$ :

$$
\frac{\partial \sigma_{x}}{\partial x}+\frac{\partial \tau_{x y}}{\partial y}=0 ; \quad \frac{\partial \sigma_{y}}{\partial y}+\frac{\partial \tau_{x y}}{\partial x}=0 ; \quad \sigma_{x}-\sigma_{y}= \pm 2\left(Z+\mu \tau_{x y}^{2} / Z\right) .
$$

Here $\sigma_{x}, \sigma_{y} \tau_{x y}$ - are normal and tangent stresses, $Z=Z(x, y)$ - is the function of the soft layer heterogeneity. In articles $[1,2,6,7]$ the function of heterogeneity was supposed to be dependent on one variable: $Z_{x y}=Z(y)$. It allowed us to reduce the system of the plastic equilibrium equations (2) under certain simplifying conditions to a combination of 
some nonlinear systems of ordinary differential equations. The approach was based on the following statement [1, p. 80, lemma $2 ; 2$, p. 86, lemma 1].

Functional equation

$$
f(x)+g(y)+f_{1}(x) g_{1}(y)=0
$$

can be solved if and only if either $f$ and $f_{1}$ are constants or $g$ and $g_{1}$ are constants.

In articles [8-10] the stressed state of the layer with the heterogeneity function of the following type has been studied for the first time:

$$
Z(x, y)=U(x) V(y) .
$$

The research into mathematical models of critical states of the plastic layer was reduced there to the solution to some nonlinear systems of the ordinary differential equations. To do this, the research into the functional equation was carried out

$$
f(x)+g(y)+f_{1}(x) g_{1}(y)+f_{2}(x) g_{2}(y)=0 .
$$

If instead of hypothesis (1) we use a more general assumption,

$$
\tau_{x y}=X(x) Y(y)+X_{1}(x) Y_{1}(y)
$$

the method used in articles [8 - 10] will require the research in the properties of solutions to the following equation:

$$
f(x)+g(y)+f_{1}(x) g_{1}(y)+f_{2}(x) g_{2}(y)+\ldots+f_{5}(x) g_{5}(y)=0 .
$$

The purpose of the paper is investigation into the properties of a more general than (5) functional equation

$$
f(x)+g(y)+f_{1}(x) g_{1}(y)+f_{2}(x) g_{2}(y)+\ldots+f_{n}(x) g_{n}(y)=0,
$$

as the basis of the development of method in papers [8 - 10] used for mathematical modeling of critical states of the plastic layers.

\section{Functional equation. Basic theorem}

Let functions

$$
f(x), f_{0}(x) \equiv 1, f_{1}(x), . ., f_{n}(x), g(y), g_{0}(y) \equiv 1, g_{1}(y), \ldots, g_{n}(y)
$$

be defined in set $\mathrm{D}$ having, at least, $n+1$ elements $(n=1,2, .$.$) and acquire values in the$ arbitrary field $F$ with zero characteristic.

Lemma 1. Let functions $f_{1}, . ., f_{n}$ be linearly independent over field F. Let $\Phi=\left(f_{i}\left(x_{j}\right)\right)-$ matrix $n \times n$. Then there exist such $x_{1}, x_{2}, \ldots x_{n} \in D$, that $\operatorname{det} \Phi \neq 0$.

Proof.

Let us suppose that this is not the case. Among all the minors of all numerical matrices obtained by substitution of the arbitrary elements from set $D$ instead of variables $x_{1}, x_{2}, \ldots x_{n}$ in matrix $\Phi=\left(f_{i}\left(x_{j}\right)\right)$, let us choose the largest in order minor $M$, being other 
than zero. Its order is presumably smaller $n$. Let us choose arbitrary minor $M_{1}$, bordering $M$, and choose in it nonintersecting minor $M$ column $f_{i}\left(x_{k}\right)$ ( parameter $i$ runs a multitude of line numbers of minor $M_{1}$ ). By the construction for every value of $x=x_{k}$ minor $M_{1}=0$. Let us expand determinant $M_{1}$ over the chosen column. We will obtain a linear combination of functions $f_{i}(x)$, with at least one of the coefficients, namely $M$, being other than zero. Hence, functions $f_{0}, f_{1}, . ., f_{n}$ are linearly dependent. This is a contradiction.

Lemma 2. Let functions $f_{0} \equiv 1, f_{1}, . ., f_{n}$ be linearly independent over field $F$. Let functions

$$
f(x), f_{1}(x), . ., f_{n}(x), g(y), g_{1}(y), \ldots, g_{n}(y)
$$

satisfy equation (6). Then functions $g(y), g_{1}(y), \ldots, g_{n}(y)$ are constant.

Proof.

According to lemma 1 , there exist such elements $x_{0}, x_{1}, \ldots x_{n}$ of set $D$ that $(n+1) \times$ $(n+1)$ determinant $\operatorname{det} \Phi\left(f_{i}\left(x_{j}\right)\right) \neq 0$.

Let us consider a system of linear equations with respect to the unknown $g, g_{1}, \ldots, g_{n}$ :

$$
f\left(x_{i}\right)+f_{0}\left(x_{i}\right) g(y)+f_{1}\left(x_{i}\right) g_{1}(y)+f_{2}\left(x_{i}\right) g_{2}(y)+\ldots+f_{n}\left(x_{i}\right) g_{n}(y)=0, \quad i=1,2, \ldots, n .
$$

The matrix determinant of this system by condition is other than zero. The only solution to this system does not depend on variable $y$, therefore it is constant: $g=$ const, $g_{1}=$ const $, \ldots, g_{n}=$ const.

Theorem 1. Let functions $f_{0} \equiv 1, f_{1}, . ., f_{k}$ be linearly independent over field $F$ and functions $f_{k+1}, \ldots, f_{n}$ linearly depend on them. Let functions (7) satisfy equation (6). Then functions $g(y), g_{1}(y), \ldots, g_{k}(y)$ linearly depend on functions $g_{0}(y) \equiv 1, g_{k+1}(y), \ldots$, $g_{n}(y)$.

Proof.

By condition,

$$
f_{s}=\sum_{i=1}^{k} \alpha_{s i} f_{i}(x), \quad s=k+1, \ldots, n .
$$

Substituting these expressions in equation (6), we obtain:

$$
\begin{aligned}
& \sum_{i=1}^{k} f_{i}(x) g_{i}(y)+\sum_{s=k+1}^{n}\left(\sum_{i=1}^{k}\left(\alpha_{s i} f_{i}(x)+\alpha_{s}\right)\right) g_{s}(y)+f+g=0, \\
& \sum_{i=1}^{k} f_{i}(x)\left(g_{i}(y)+\sum_{s=k+1}^{n} \alpha_{s i} g_{s}(y)\right)+\sum_{s=k+1}^{n} \alpha_{s} g_{s}(y)+f+g=0 .
\end{aligned}
$$

Since by condition functions $f_{i}, i=0,1, \ldots, k$, are linearly independent, from lemma 2 it is evident that

$$
g_{i}(y)+\sum_{s=k+1}^{n} \alpha_{s i} g_{s}(y)=c_{i} ; i=1, \ldots, k
$$




$$
\sum_{s=k+1}^{n} \alpha_{s} g_{s}(y)+g=c .
$$

Hence, functions $g(y), g_{1}(y), \ldots, g_{k}(y)$ linearly depend on functions $g_{0}(y) \equiv 1, g_{k+1}(y)$, $\ldots, g_{n}(y)$.

We can suggest another statement of theorem 1.

Theorem 2. Let there be $r_{f}$ of linearly independent functions over field $F$ among functions $f_{0} \equiv 1, f_{1}, \ldots, f_{n}$, there is $r_{g}$ of linearly independent functions over field $F$ among functions $g, g_{0}, . . g_{n}$ and functions

$$
f(x), f_{1}(x), . ., f_{n}(x), g(y), g_{1}(y), \ldots, g_{n}(y)
$$

satisfy equation (6). Then $r_{f}+r_{g} \leq n+2$.

From the viewpoint of applications to heterogeneous plastic media, of particular interest is case $n=2$, i.e. equation (5).

Theorem 3. With $n=2$ the following cases are realized.

Case 1: $r_{f}+r_{g}=2$. Then all the functions

$$
f(x), f_{1}(x), f_{2}(x), g(y), g_{1}(y), g_{2}(y)
$$

are constant. These constants have to satisfy equation (5):

$$
f+g+f_{1} g_{1}+f_{2} g_{2}=0
$$

Case 2: $r_{f}+r_{g}=3$. Let $r_{f}=1, r_{g}=2$. Then functions

$$
f(x), f_{1}(x), f_{2}(x)
$$

are constant. Functions

$$
g(y), g_{1}(y), g_{2}(y)
$$

are not constant. For some elements of field $F \alpha, \alpha_{1}, \alpha_{2}, \alpha^{2}+\alpha_{1}^{2}+\alpha_{2}^{2} \neq 0$ the following equations holds:

$$
\alpha g(y)+\alpha_{1} g_{1}(y)+\alpha_{2} g_{2}(y)=0
$$

as well as equation (5)

$$
f+g(y)+f_{1} g_{1}(y)+f_{2} g_{2}(y)=0 .
$$

Functions $g_{1}(y) u g_{2}(y)$ depend linearly on $g(y)$. If $r_{f}=2, r_{g}=1$, then functions

$$
g(x), g_{1}(x), g_{2}(x)
$$

are constant. This case is symmetrical to the previous one.

Case 3: $r_{f}+r_{g}=4$. If $r_{f}=1, r_{g}=3$, then functions $f(x), f_{1}(x), f_{2}(x)$ are constant. This variant was considered in case 1 . Let $r_{f}=2, r_{g}=2$. Then two subcases are possible.

Case 3.1. There exist $\alpha, \beta \neq 0, \gamma, \delta \in R$ such that

$$
f_{2}(x)=\alpha+\beta f_{1}(x)
$$




$$
\begin{gathered}
f(x)+\delta f_{1}(x)+\gamma=0, \\
g(y)=\alpha g_{2}(y)=\gamma, \\
g_{1}(y)+\beta g_{2}(y)=\delta .
\end{gathered}
$$

Case 3.2. There exist $\alpha \neq 0, \beta, \gamma \in R$ such that

$$
\begin{gathered}
f_{1}(x) \equiv \alpha, \\
f(x)+\beta f_{2}(x)+\gamma=0, \\
g_{2}(y)=\beta, \\
g(y)+\alpha g_{1}(y)=\gamma .
\end{gathered}
$$

\section{Example of reducing system (2) to a system of ordinary differential equations}

The result of system (2) under conditions (1) and (3) is equation [8, 9]:

$$
\left(2 U^{\prime} / X\right)\left(2 V^{\prime} / Y\right)-(1 / X)\left(X^{2} / U\right)^{\prime}(1 / Y)\left(Y^{2} / V\right)^{\prime}+X^{\prime \prime} / X-Y^{\prime \prime} / Y=0 .
$$

Suppose, using designations from $(1),(4)$ and (5),

$$
\begin{gathered}
f(x)=U^{\prime} / X, g(y)=2 V^{\prime} / Y, f_{1}(x)=-(1 / X)\left(X^{2} / U\right)^{\prime}, \\
g_{1}(y)=(1 / Y)\left(Y^{2} / V\right)^{\prime}, f_{2}(x)=X^{\prime \prime} / X, g_{2}(y)=-Y^{\prime \prime} / Y .
\end{gathered}
$$

Then each of the three mentioned cases of theorem 3 results in the system of ordinary differential equations. As an example, let us consider the case when all the 6 values of $g, g_{1}, g_{2}, f, f_{1}, f_{2}$ are constant. Then the following system of equations with an independent variable $y$ occurs:

$$
V^{\prime} / Y=g,(1 / Y)\left(Y^{2} / V\right)^{\prime}=g_{1}, Y^{\prime \prime} / Y=g_{2},
$$

as well as the similar system with variable $x$. Solution to system (9) with initial conditions (7) yields three cases depending on the sign of constant $g_{2}$. Suppose $g_{2}=-a^{2}, a \neq 0$. Then from (9) follows equation

$$
Y^{\prime \prime}+a^{2} Y=0
$$

Solution to this equation under condition (7) is function

$$
Y=C_{1} \sin (a y)
$$

where $C_{1}$ is an arbitrary constant. Using the other equations of system (9), we can obtain

$$
\left.a=\sqrt{-g g_{1}}, \quad V=0.5\left(1+\cos \sqrt{-g g_{1}} y\right), \quad Y=0.5 \sqrt{-g_{1} / g} \sin \sqrt{g g_{1}} y\right) .
$$

If $g_{2}=a^{2}, a \neq 0$, we can obtain similarly:

$$
V=0.5\left(1+\operatorname{ch}\left(\sqrt{g g_{1}} y\right)\right), Y=0.5 \sqrt{g_{1} / g} \operatorname{sh}\left(\sqrt{g g_{1}} y\right) .
$$

Thus we get similar expressions of the second system of equations for the functions depending on $x$. Depending on the increase or decrease of functions $U, V$, four variants of solution to equation (6) are obtained. For example, if functions $U(x), V(y)$ are decreasing, then

$$
Z(x, y)=U(x) V(y)=0.25\left(1+\cos \sqrt{-g g_{1}} y\right)\left(1+\cos \sqrt{-f f_{1}} x\right) .
$$

Tangent stresses in the layer are calculated according to formula (1). Using equations of equilibrium and the condition of plasticity (2), by integration we can get analytical expressions for calculating normal stresses and critical load. 


\section{References}

1. Dilman V.L., Eroshkina T.V. [Mathematical Modelling of Critical States of the Soft Layers in Heterogeneous Joints]. Chelyabinsk, Publishing Center of South Ural State University, 2011. (in Russian)

2. DilmanV.L. [Mathematical Models of the Stress State of Heterogeneous Thin-walled Cylindrical Shells]. Chelyabinsk, Publishing Center of South Ural State University, 2007. (in Russian)

3. Dilman V.L., Ostsemin A.A. [Stress State and Static Strength of the Plastic Interlayer in Plane Deformation]. Journal of Machinery Manufacture and Reliability, 2005, no. 4, pp. 38-48. (in Russian)

4. Dilman V.L., Ostsemin A.A. [On the Stress-deformed State at the Tension of the Plastic Layer with Two Axes of Symmetry]. Mechanics of Solids, 2001, no. 6, pp. 115124 (in Russian)

5. Dilman V.L., Nosachova A.A. Mathematical Simulation of Critical States of the Plastic Layer]. Tambov University Reports. Series: Natural and Technical Sciences, 2013, vol. 18, issue 5-2, pp. 2502-2504. (in Russian)

6. DilmanV.L. [The Stress State of the Plastic Layer with Variable Strength Broadwise]. Mechanics of Solids, 2000, no. 1, pp. 141-148. (in Russian)

7. Dilman V.L. Research of the Mathematical Models of the Stress Condition of the Thin-walled Heterogeneous Cylindrical shells Based on Analytical Methods]. Bulletin of the South Ural State University. Series "Mathematical Modelling, Programming 85 Computer Software", 2009, issue 3, no. 17(150), pp. 36-58. (in Russian)

8. Dilman V.L., Trunova D.A. [Mathematical Modeling of the Critical Sates of a Heterogeneous Plastic Rectangle at Plane Deformation]. Bulletin of the Magnitogorsk State University. Mathematics, 2012, issue 14, pp. 46-55. (in Russian)

9. Dilman V.L., Trunova D.A. [Mathematical Modelling of the Critical States of the Heterogeneous Layer Under Different Conditions of Heterogeneity]. Molodoy Issledovatel' - Materialy 66-y Studencheskoy Nauchnoy Konferentsii. [Young Researcher - Proceedings of 66-th Student Scientific Conference]. Chelyabinsk, Publishing center of SUSU, 2013, vol. 2, pp. 166-171. (in Russian)

10. Dilman V.L., Trunova D.A. [The Stress State of a Rectangular Plastic Layer with the Function of Heterogeneity Depending on Two Variables]. Yuzhno-Uralskaya Molodezhnaya Shkola po Matematicheskomu Modelirovaniyu, Chelyabinsk, 29-30 Maya 2014. [South Ural Youth School on Mathematical Modeling, Chelyabinsk, 29-30 May 2014]. Chelyabinsk, Publishing center of SUSU, 2014, pp. 144-151. (in Russian)

Valery L. Dilman, Doctor of Physico-Mathematical Sciences, Docent, Head of the Department of Applied Mathematics, South Ural State University, Chelyabinsk, Russian Federation, dilman49@mail.ru

Darya A. Trunova, Postgraduate student, Department of Applied Mathematics, South Ural State University, Chelyabinsk, Russian Federation, jessica192@mail.ru

Received May 13, 2015 